\title{
Capillary electrophoresis of biomolecules
}

\author{
Lisa Holland ${ }^{1}$
}

Received: 17 July 2015 / Accepted: 17 July 2015 / Published online: 31 July 2015

(C) Springer-Verlag Berlin Heidelberg 2015

The introduction of modern capillary electrophoresis to separation science has dramatically changed the nature of bioanalytical research. Selectivity based on charge-to-size ratio can be expanded with the addition of secondary selectors to the background electrolyte. The ability to quickly change the separation mechanism to size, affinity binding, partitioning, ion pairing, or complexation is the driving force for bioanalyses with capillary electrophoresis. Separations are typically completed within minutes and generate efficiencies ranging from tens of thousands to millions of theoretical plates. When automated, capillary electrophoresis is well suited for high-throughput assays. As a miniaturized technique, microchip capillary electrophoresis systems perform under extraordinary conditions. The goal of this topical collection on capillary electrophoresis applied to biomolecule separations is to demonstrate the wide range of established and emerging applications of this powerful method. An overview of three unique areas of interest is communicated through critical reviews on DNA analyses, clinical biomarkers, and remote operation on future spaceflight missions.

Capillary electrophoresis is an enabling tool for DNA separation. It is an established method to size DNA for pathogen, animal, and human identification. The field continues to advance as evidenced through the new strategies reported in this collection to preferentially extract DNA from specific cells as a means to improve analyses of critical forensic samples. An

Published in the topical collection Capillary Electrophoresis of Biomolecules with guest editor Lisa Holland.

Lisa Holland

lisa.holland@mail.wvu.edu

1 C. Eugene Bennett Department of Chemistry, West Virginia University, 217 Clark Hall, Morgantown, WV 26506, USA evolving area of capillary electrophoresis is the selection of molecular binding elements comprised of single-stranded DNA using computational tools and the method of systematic evolution of ligands by exponential enrichment. A paper in this issue by Eaton et al. demonstrates the utility of capillary electrophoresis and bioinformatics for the generation of an aptamer for the HE4 ovarian cancer biomarker. High-throughput parallel sequencing when combined with electrophoretic selection of aptamers provides insight into synthesis bias of the combinatorial library and artifacts introduced through amplification or preparatory steps for parallel sequencing.

Capillary electrophoresis separations typically consume only nanoliter sample volumes; however, such a small injection volume can prevent analyses requiring low detection limits. Two different strategies to increase mass loading are described in this issue. The report on circulating steroids by Nyakubaya et al. utilizes field amplification and $\mathrm{pH}$-mediated stacking to achieve nanomolar detection limits with UV-visible absorbance. This approach separates six steroidal compounds in $5 \mathrm{~min}$ in a plasma volume at or below $5 \mu \mathrm{L}$. With a stacking based on isotachophoresis reported by Phun et al., whole Escherichia coli cells can be detected at a concentration as low as 78 cells $/ \mathrm{mL}$. This is a striking improvement over the method of plate counting estimated to generate a detection limit of $100,000 \mathrm{cells} / \mathrm{mL}$.

Microfluidics and microchips play a crucial role in realizing point-of-care measurements of biomarkers, including lipids, small molecules, nucleic acids, proteins, and specific cells. A review of recent developments in this area by Pagaduan et al. highlights applications with different detection strategies, device design, and fabrication materials. The technology that supports assays for these clinical biomarkers can also provide powerful insight into fundamental biochemical processes. As an example in this special issue, de Campos et al. used a hybrid glass-PDMS chip to monitor superoxide production in stimulated macrophage cells. These electrophoretic separations 
improve superoxide analyses by distinguishing a fluorescent superoxide conjugate from fluorescent products generated by oxidizing interferences present in the cellular sample.

The diversity in biomolecular analyses and the compatibility with lab-on-a-chip technology are uniquely suited to search for molecular signatures of life. In this issue, Willis et al. describe capillary electrophoresis strategies to identify nonstatistical distributions of organic molecules, such as homology and chirality. These remote analyses have extreme constraints on the analytical measurement, including effects of gravity, pressure, temperature, and true analytical device autonomy from sample processing to detection of labeled products. The articles in this topical collection reveal how capillary electrophoresis continues to transform bioanalytical research especially under challenging settings such as space flight. I sincerely thank the editors and editorial staff for the opportunity to share this progress in analytical separations.

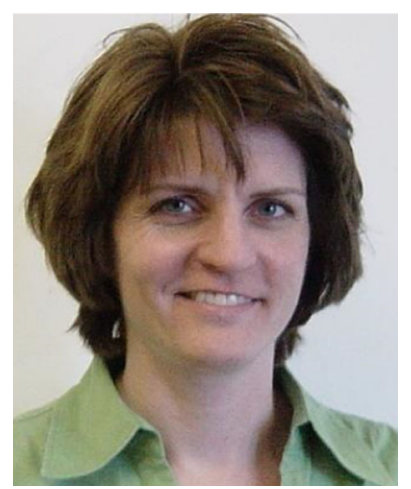

Lisa Holland is a Professor in the C. Eugene Bennett Department of Chemistry at West Virginia University, specializing in bioanalytical microscale separations. Her research interests are in multifunctional nanomaterials to both process and sort biomolecules, microscale sequencing of linear and branched oligosaccharides, high-throughput profiling of biomarkers of endocrine disruption, and rapid screening of nanoparticle-biomolecule affinity. 